\title{
A Hopf-Lax Formula for the Level-Set Equation and Applications to PDE-Constrained Shape Optimisation
}

\author{
Daniel Kraft \\ University of Graz \\ Institute of Mathematics, NAWI Graz \\ Universitätsplatz 3, 8010 Graz, Austria \\ Email: daniel.kraft@uni-graz.at
}

\begin{abstract}
Level-sets are a flexible method to describe geometries and their changes according to a speed field. This can be used in a wide variety of applications. We will present a HopfLax formula that can be used to represent the solution of the level-set equation as well as the described geometries directly. This formula is a generalisation of existing results to the case of speed fields without a uniform, positive lower bound. The corresponding equation is of Hamilton-Jacobi type with a nonconvex Hamiltonian.

Our representation formula can be used both for theoretical and numerical purposes. In the latter case, the Fast Marching Method can be applied, leading to very efficient and robust numerical calculations of the geometry evolutions. We will also apply the level-set framework to an illustrative problem in PDEconstrained shape optimisation, and present numerical results.
\end{abstract}

\section{INTRODUCTION}

In the context of shape optimisation, an important issue is the description of geometric variables (i. e., the shape of some domain) in terms of objects that are easier to handle within a mathematical framework. Different classical possibilities to describe such geometrical situations can be found in chapter 2 of [1]. A lot of literature about shape optimisation is dedicated to the investigation of methods based on the transformation of the studied geometry onto a reference domain. See, for instance, [2] and [3] for extensive discussions of these types of methods.

An alternative approach is the use of level-set methods as introduced in [4] and analysed, for instance, in [5] extensively. The basic idea is to use a function $\phi: \mathbb{R}^{n} \rightarrow \mathbb{R}$ to describe a domain $\Omega$ via its zero level-set, i. e.,

$$
\Omega=\phi^{-1}((\infty, 0))=\left\{x \in \mathbb{R}^{n} \mid \phi(x)<0\right\} .
$$

Assuming that $\phi$ is at least continuous, $\Omega$ defined in this way is an open set. In the following, we will always denote the boundary of such a domain by $\Gamma=\partial \Omega$. One usually expects that the zero level-set of $\phi$ is not "fat", meaning that

$$
\Gamma=\partial \Omega=\phi^{-1}(\{0\})
$$

holds. The problem of "fattening" is treated (including sufficient conditions for this property to hold true) in [6].
For changes of the geometry of $\Omega$, we will use a levelset variant of the classical speed method: The "direction" of change can be characterised by a speed field $F: \mathbb{R}^{n} \rightarrow \mathbb{R}$. For any $x \in \mathbb{R}^{n}$, the value $F(x)$ describes the speed with which the boundary of $\Omega$ moves in normal direction at this point. Positive $F$ means outward movement, negative $F$ inward. $F=0$ corresponds to parts of the geometry that do not change. In the level-set framework, these changes in geometry are achieved by making $\phi$ time-dependent. In particular, the time evolution of $\phi$ matching the desired changes in geometry according to a speed field $F$ is governed by the level-set equation

$$
\phi_{t}(x, t)+F(x)|\nabla \phi(x, t)|=0
$$

together with a suitable initial condition $\phi(x, 0)=\phi_{0}(x)$. Here, $\phi_{0}$ is a level-set function that describes the initial geometry $\Omega_{0}$. Often, $\phi_{0}$ is chosen as the signed distance function of $\Omega_{0}$. We will assume in the following that both $\phi_{0}$ and $F$ are Lipschitz continuous. For $\phi_{0}$, this can be ensured by choosing it indeed as a signed distance function. Furthermore, we will also assume that $F$ has compact support, since this is no practical restriction.

This equation can already be found as (2.13) in [4]. It is of Hamilton-Jacobi type, and the proper concept of solutions for (1) is that of viscosity solutions. See, for instance, [7] and [8]. For the sake of completeness, we will quickly recall the definition:

Definition 1. Let $D=\mathbb{R}^{n} \times(0, \infty)$ be the open space-time cylinder, $\phi: D \rightarrow \mathbb{R}$ and $(x, t) \in D$. Then $J^{1+} \phi(x, t)$ is the set of all $(p, a) \in \mathbb{R}^{n} \times \mathbb{R}$ such that

$\phi(y, s) \leq \phi(x, t)+a(s-t)+p \cdot(y-x)+o(|s-t|+|y-x|)$ as $(y, s) \rightarrow(x, t)$ in $D$. Similarly, $(p, a) \in J^{1-} \phi(x, t)$ if and only if

$\phi(y, s) \geq \phi(x, t)+a(s-t)+p \cdot(y-x)+o(|s-t|+|y-x|)$

for $(y, s) \rightarrow(x, t) . J^{1 \pm} \phi(x, t)$ are called the first-order parabolic semijets of $\phi$ at $(x, t)$. Note that $J^{1-} \phi(x, t)$ is often also called subdifferential of $\phi$ at $(x, t)$. 
Definition 2. Let $F$ and $\phi_{0}$ be given. We say that $\phi: \mathbb{R}^{n} \times$ $[0, \infty) \rightarrow \mathbb{R}$ is a viscosity subsolution of (1) for the given data if $\phi$ is upper semi-continuous, $\phi(\cdot, 0) \leq \phi_{0}$ on $\mathbb{R}^{n}$ and

$$
a+F(x)|p| \leq 0
$$

for each $x \in \mathbb{R}^{n}, t>0$ and $(a, p) \in J^{1+} \phi(x, t)$. Similarly, $\phi$ is a viscosity supersolution if $\phi$ is lower semi-continuous, $\phi(\cdot, 0) \geq \phi_{0}$ and $a+F(x)|p| \geq 0$ for all $(a, p) \in J^{1-} \phi(x, t)$.

$\phi$ solves (1) in the viscosity sense if $\phi$ is both a viscosity sub- and supersolution. Note that this implies in particular that $\phi$ is continuous and that $\phi(x, 0)=\phi_{0}(x)$ for all $x \in \mathbb{R}^{n}$.

It is well-known that (1) has a unique solution $\phi: \mathbb{R}^{n} \times$ $[0, \infty) \rightarrow \mathbb{R}$ in this sense for any given (continuous) $F$ and $\phi_{0}$. Furthermore, the so-called comparison principle holds: If $\phi_{1}$ and $\phi_{2}$ are a viscosity sub- and supersolution to (1), respectively, with $\phi_{1}(\cdot, 0) \leq \phi_{2}(\cdot, 0)$ on $\mathbb{R}^{n}$, then $\phi_{1} \leq \phi_{2}$ on $\mathbb{R}^{n} \times[0, \infty)$. For the proofs, see, for instance, [7] or [5].

\section{THE Hopf-LAX Formula}

In this section, we will state and motivate our main result. The technical details of the proofs will not be given here, they will be published in a forthcoming paper. We will assume $F \geq 0$, since by using appropriate symmetry properties of the solution $\phi$ of (1), one can reduce the general case to the case of non-negative speeds. Furthermore, it can be shown that $\phi(x, t)=\phi_{0}(x)$ for all $t \geq 0$ whenever $F(x)=0$. Thus, the main focus of our investigation will be the set $\Omega^{+}=$ $F^{-1}((0, \infty))$. This set is open and bounded, since we assumed that $F$ is Lipschitz continuous and compactly supported.

\section{A. Shortest Paths}

Instead of the time-dependent Cauchy problem (1), we start by considering the stationary Eikonal equation

$$
F(x)\left|\nabla d_{y}(x)\right|=1, \quad d_{y}(y)=0
$$

for some fixed source point $y \in \Omega^{+}$. We consider this equation on the connected component $C$ of $\Omega^{+}$containing $y$. The solution to (2) is understood again in the viscosity sense similar to Definition 2 above. Intuitively, $d_{y}(x)$ gives the "distance" (induced by the speed field $F$ ) between two points $x$ and $y$. This connection can also be formalised by considering paths between $y$ and other points $x \in C$ :

Definition 3. Let $x, x^{\prime} \in C$. A path connecting $x$ and $x^{\prime}$ is a function $\xi \in W^{1, \infty}([0,1], C)$ with $\xi(0)=x$ and $\xi(1)=x^{\prime}$. $X_{\mathrm{ad}}\left(x, x^{\prime}\right)$ is the set of all such paths. For $\xi \in X_{\mathrm{ad}}\left(x, x^{\prime}\right)$, the $F$-induced length of $\xi$ is defined as

$$
l(\xi)=\int_{0}^{1} \frac{\left|\xi^{\prime}(t)\right|}{F(\xi(t))} d t .
$$

Note that by the Sobolev embedding theorem (see, for instance, Theorem 6 on page 270 of [9]), each such path $\xi \in X_{\text {ad }}\left(x, x^{\prime}\right)$ is continuous. Furthermore, $F \circ \xi$ attains a strictly positive minimum on $[0,1]$, such that $(3)$ is welldefined with $0 \leq l(\xi)<\infty$.
Definition 4. For $x \in \mathbb{R}^{n}$, we set $d(x, x)=0$. For $x^{\prime} \neq x$, if there exist paths in $X_{\text {ad }}\left(x, x^{\prime}\right)$, define

$$
d\left(x, x^{\prime}\right)=\inf _{\xi \in X_{\text {ad }}\left(x, x^{\prime}\right)} l(\xi)
$$

as the shortest distance. Otherwise, set $d\left(x, x^{\prime}\right)=\infty$.

Note that this notion is already used in [10], although there it is assumed that $F$ is uniformly bounded away from zero. Given Definition 3 and Definition 4, the following basic properties of this distance can be shown easily:

Lemma 1. $d(\cdot, \cdot)$ induces a metric on $\mathbb{R}^{n}$ and when restricted to a connected component of $\Omega^{+}, d(\cdot, \cdot)$ is continuous in both arguments.

We can now state the first main result, which connects this distance of shortest paths to the viscosity solution of the stationary Eikonal equation (2):

Theorem 1. Let $C \subset \Omega^{+}$be a connected component and $y \in C$ be fixed. Then $d_{y}(\cdot)=d(\cdot, y)$ is a viscosity solution of (2) in $C \backslash\{y\}$.

Proof: For a given cut-off threshold $\underline{F}>0$, we define an auxiliary speed $\tilde{F}(x)=\max (\underline{F}, F(x))$ and the corresponding distance $d_{F}$ induced by $\tilde{F}$. For these distances, the result is well-known (Theorem 5.1 on page 117 of [10]). Thus it remains to verify that the claim stays true even if we remove the cut-off.

Let $x \in C$ be fixed. The crucial point is to notice that there exists a threshold $\underline{F}>0$ such that only paths $\xi \in X_{\text {ad }}(x, y)$ with $F(\xi(t)) \geq \underline{F}$ for all $t \in[0,1]$ are interesting for the infimum in Definition 4. This is the case due to Lipschitz continuity of $F$. Thus, $d(x, y)=d_{F}(x, y)$ can be achieved in a neighbourhood of $x$. Since being a viscosity solution is a local property, this yields with some further considerations that the result of [10] can indeed be applied to show that $d(\cdot, y)$ solves (2).

\section{B. The Hopf-Lax Formula}

Theorem 2. Let $F \geq 0$ and $d(\cdot, \cdot)$ be the distance introduced in Definition 4. Then the viscosity solution to (1) is given by the Hopf-Lax formula

$$
\phi(x, t)=\inf \left\{\phi_{0}(y) \mid y \in \mathbb{R}^{n}, d(x, y) \leq t\right\}
$$

for all $t \geq 0$ and $x \in \mathbb{R}^{n}$.

Proof: For the case of $F$ uniformly positive, this follows from Theorem 3.1 on page 140 of [11]. It can be generalised to the case $F \geq 0$ using the same arguments as in the proof of Theorem 1 .

Using this result, one can also deduce a corresponding formula for the geometries $\Omega_{t}$ themselves represented by $\phi(\cdot, t)$. Let $\Omega_{0}$ be an initial domain. Since $F \geq 0$ by assumption, the initial geometry moves only outward and thus $\Omega_{t}$ grows in time. 
Definition 5. Let $d(\cdot, \cdot)$ be the distance of Definition 4 as before. Then, for $x \in \mathbb{R}^{n}$, we define

$$
d_{0}(x)=\inf _{y \in \Omega_{0}} d(x, y) .
$$

For each $x \in \mathbb{R}^{n}, d_{0}(x) \in[0, \infty]$ is the $F$-induced distance to the initial set. One can show that $d_{0}$ is locally Lipschitz continuous and solves the Eikonal equation

$$
F(x)\left|\nabla d_{0}(x)\right|=1
$$

for almost-all $x \in \Omega^{+} \backslash \overline{\Omega_{0}}$. In other words, $d_{0}(x)$ is the time until the evolving geometry hits $x$. This intuitive understanding can easily be formalised to yield:

Theorem 3. Let $F \geq 0$ and $\Omega_{t}$ be the time evolution of some initial geometry $\Omega_{0}$ according to the level-set method and (1). Then

$$
\Omega_{t}=d_{0}^{-1}((-\infty, t))=\left\{x \in \mathbb{R}^{n} \mid d_{0}(x)<t\right\}
$$

holds for all $t>0$.

It is important to realise that the representation found in Theorem 3 can be used for numerical purposes: The quantity $d_{0}$ of Definition 5 can be calculated efficiently using the Fast Marching Method [12]. Even if $F \geq 0$ does not hold throughout the domain, one can split the domain into a part with $F \geq 0$ and one with $F \leq 0$ and apply Fast Marching separately to each part. When this is done, one can "concatenate" the parts and use Theorem 3 to construct a levelset function for the propagating geometry at arbitrary times $t \geq 0$. This "composite Fast Marching Method" can thus be used to evolve a geometry in time for arbitrary speed fields and does not require the front to move monotonically outward. Furthermore, if, for instance, a line search is done, one can even use the same $d_{0}$ to test multiple "step lengths" (evolution times $t$ in this case). This reduces computation times even further.

\section{Shape Derivatives}

In addition to its usability from a numerical point of view, Theorem 3 also allows to draw theoretical consequences. One of particular interest is the shape derivative of a domain functional, which we want to sketch here quickly. For this, let $f \in L_{\text {loc }}^{1}\left(\mathbb{R}^{n}\right)$. We consider

$$
J(t)=\int_{\Omega_{t}} f d x
$$

Theorem 4. Let $\Gamma_{0}$ be a null set and $F \geq 0$. Then,

$$
J(t)=J(0)+\int_{0}^{t} \int_{d_{0}-1(\{s\})} F f d \sigma d s
$$

holds for $t \geq 0$. As an immediate consequence, $J$ is differentiable for almost-all $t \geq 0$ with

$$
J^{\prime}(t)=\int_{d_{0}^{-1}(\{t\})} F f d \sigma .
$$

Proof: The derivative result follows immediately by the Lebesgue differentiation theorem once the representation of $J$ itself is shown. For $t=0$, the claim is clear. Assume $t>0$ from now on. Take note that

$$
J(t)=J(0)+\int_{\Omega_{t} \backslash \overline{\Omega_{0}}} f d x
$$

since $\Omega_{0} \subset \overline{\Omega_{0}} \subset \Omega_{0} \cup \Gamma_{0}$ and all these sets differ by at most $\Gamma_{0}$ which has measure zero. Furthermore, Theorem 3 allows us to write

$$
\Omega_{t} \backslash \overline{\Omega_{0}}=\Omega^{+} \cap d_{0}^{-1}((0, t))
$$

Recall also (4), which applies to this set.

Next, let $\chi$ be the characteristic function of $d_{0}{ }^{-1}((0, t))$ and define $g=\chi F f$. Then $g \in L^{1}\left(\mathbb{R}^{n}\right)$ since $F$ has compact support. The result now follows from the co-area formula (Theorem 2 on page 117 of [13]), since

$$
\begin{aligned}
& \int_{\Omega^{+} \cap d_{0}^{-1}((0, t))} f d x=\int_{\mathbb{R}^{n}}\left|\nabla d_{0}\right| g d x \\
& =\int_{\mathbb{R}} \int_{d_{0}^{-1}(\{s\})} \chi F f d \sigma d s=\int_{0}^{t} \int_{d_{0}-1(\{s\})} F f d \sigma d s .
\end{aligned}
$$

To get around the fact that $d_{0}$ is only locally Lipschitz continuous, one can introduce compact cut-offs and use the dominated convergence theorem.

Again, using appropriate symmetry properties, one can easily generalise these results for Lipschitz continuous $F$ without the restriction $F \geq 0$.

\section{PDE-CONSTRAINED Shape OPTIMISATION}

We want to demonstrate how the level-set framework can be used for PDE-constrained shape optimisation. For this goal, we consider a simple elliptic problem with a tracking-type cost functional. Let $D \subset \mathbb{R}^{2}$ be compact, $B \subset D, f \in L^{2}(D)$ and $u_{d} \in L^{2}(B)$. Consider a sufficiently regular domain $\Omega$ with $B \subset \Omega \subset D$ and let $u=u(\Omega)$ satisfy the state equation

$$
\left\{\begin{array}{rll}
-\Delta u+u & =f & \text { in } \Omega, \\
\frac{\partial u}{\partial \nu} & =0 & \text { on } \Gamma=\partial \Omega .
\end{array}\right.
$$

We want to minimise

$$
J(\Omega)=\frac{1}{2}\left\|u-u_{d}\right\|_{L^{2}(B)}^{2}+R(\Omega)
$$

with respect to the domain $\Omega . R(\Omega)$ is a regularisation term, and we will use

$$
R(\Omega)=\alpha|\partial \Omega|=\alpha|\Gamma|
$$

with $\alpha \geq 0$ in the following.

For simplicity, all calculations presented below will be only formal. We will assume without further notice that $u$ and $\Omega$ have all the necessary regularity properties. 


\section{A. Shape Derivatives}

We have already seen that a "direction" of change of $\Omega$ can be represented in the level-set approach via a speed field $F \in C^{0,1}(D)$. As before, we denote the time evolution of some initial domain $\Omega_{0}$ in some direction $F$ according to the level-set equation (1) by $\Omega_{t}$, where $t \geq 0$. The formal shape derivative of $J$ in direction $F$ is given by

$$
\begin{aligned}
d J\left(\Omega_{0} ; F\right) & =\lim _{t \rightarrow 0^{+}} \frac{J\left(\Omega_{t}\right)-J\left(\Omega_{0}\right)}{t} \\
& =\int_{B}\left(u-u_{d}\right) \cdot u^{\prime}(x ; F) d x+d R\left(\Omega_{0} ; F\right) .
\end{aligned}
$$

Here, $u^{\prime}(\cdot ; F)$ denotes the shape derivative of the state $u$. We assume that it exists and is sufficiently regular. The shape derivative of the regularisation term can be expressed in terms of the mean curvature $\kappa$ as

$$
d R\left(\Omega_{0} ; F\right)=\int_{\Gamma_{0}} \alpha \kappa F d \sigma
$$

See, for instance, section 2.33 of [2] for this well-known result.

The next step is to calculate $u^{\prime}(\cdot ; F)$. For this, we consider the weak form of the state equation (5),

$$
\int_{\Omega}(\langle\nabla u, \nabla v\rangle+u v) d x=\int_{\Omega} f v d x
$$

for all test functions $v \in H^{1}(\Omega)$. Without loss of generality, we can also consider $v \in H^{1}(D)$ since $\Omega \subset D$ is always the case. Taking now the shape derivative of both sides of (6) using Theorem 4 , we get

$$
\begin{aligned}
& \int_{\Gamma}(\langle\nabla u, \nabla v\rangle+u v) F d \sigma \\
& +\int_{\Omega}\left(\left\langle\nabla u^{\prime}(x ; F), \nabla v\right\rangle+u^{\prime}(x ; F) v\right) d x=\int_{\Gamma} f v F d \sigma
\end{aligned}
$$

for all $v \in H^{1}(D)$. Thus, given a direction $F$, one can evaluate $u^{\prime}(\cdot ; F)$ by solving the variational problem (7).

Having to solve such a problem for each $F$ of interest separately is, however, rather costly. An alternative is the adjoint approach. For a general discussion about this classical technique, see, for instance, chapter 3 of [3]. In our case, assume we define the adjoint state $p \in H^{1}(\Omega)$ satisfying

$$
\int_{\Omega}(\langle\nabla p, \nabla w\rangle+p w) d x=\int_{B}\left(u-u_{d}\right) w d x
$$

for all $w \in H^{1}(\Omega)$. With (8) and (7), we can then express the first term of $d J(\Omega ; F)$ as

$$
\begin{aligned}
\int_{B}(u- & \left.u_{d}\right) \cdot u^{\prime}(x ; F) d x \\
& =\int_{\Omega}\left(\left\langle\nabla p, \nabla u^{\prime}(x ; F)\right\rangle+p \cdot u^{\prime}(x ; F)\right) d x \\
& =\int_{\Gamma}(f p-\langle\nabla u, \nabla p\rangle-u p) F d \sigma .
\end{aligned}
$$

Taking everything together, we have shown that the shape derivative in some direction $F$ can be expressed as:

$$
d J(\Omega ; F)=\int_{\Gamma}(f p-\langle\nabla u, \nabla p\rangle-u p+\alpha \kappa) F d \sigma
$$

In this formulation, we only have to solve the state and adjoint equations once for some geometry $\Omega$. Afterwards, $d J(\Omega ; F)$ can be evaluated for arbitrary directions $F$ simply by integrating over $\Gamma$.

\section{B. A Gradient-Descent Method}

Building on the results of the previous subsection, we will now describe a possible gradient-descent method for shape optimisation of our example problem. Since we already know how to calculate the shape derivative in some direction $F$, it remains to find a way to calculate a good descent direction. To this end, consider

$$
\min _{F} d J(\Omega ; F), \quad\|F\|_{H^{1}(D)}=1 .
$$

By using the Lagrangian for this problem, one can show that the minimising speed field $F$ is, up to normalisation, the solution of the variational problem

$$
\left\langle F^{\prime}, G\right\rangle_{H^{1}(D)}=\int_{D}\left(\left\langle\nabla F^{\prime}, \nabla G\right\rangle+F^{\prime} G\right) d x=\int_{\Gamma} a G d \sigma
$$

for all $G \in H^{1}(D)$. Here,

$$
a=f p-\langle\nabla u, \nabla p\rangle-u p+\alpha \kappa
$$

denotes the expression appearing in the trace-integral form of $d J(\Omega ; F)$ according to (9). In other words, we look for the Riesz representative $F^{\prime}$ of the shape derivative $d J(\Omega ; \cdot)$. This is, per definition, the shape gradient and thus $-F^{\prime}$ is a prime candidate for a descent direction.

Furthermore, we have

$$
\left\|F^{\prime}\right\|_{H^{1}(D)}^{2}=\int_{\Gamma} a F^{\prime} d \sigma=d J\left(\Omega ; F^{\prime}\right)=\lambda,
$$

which can be useful to determine how close a given geometry is already to a stationary point with vanishing derivative. Finally, the solution $F$ of (10) is then simply $F=-\frac{1}{\lambda} F^{\prime}$. In order to ensure that $B \subset \Omega \subset D$ is always the case, one can set the speed to zero on $B \cap F^{-1}((-\infty, 0))$ and $D \cap F^{-1}((0, \infty))$ to prevent shrinking into $B$ or growing outside of $D$.

One can also easily introduce an additional weighting factor $\beta>0$ and use

$$
\langle F, G\rangle_{\beta}=\int_{D}(F G+\beta\langle\nabla F, \nabla G\rangle) d x
$$

instead of the ordinary metric in $H^{1}(D)$. Usually, one wants the speed field to be smoothed in normal direction but allow for variations along $\Gamma$. By choosing $\beta \ll 1$, one can achieve some smoothing but also allow more variations than for $\beta=1$. This was beneficial for some of our calculations. See Subsection IV-B for a demonstration of this effect.

\section{NumERICAL RESUlts}

In this section, we want to present the results of our methods applied to two test cases for our shape optimisation problem. They were constructed by defining a forcing function $f$ as well as a "precise" initial shape. For the first example, the exact solution $u_{d}$ of (5) is known analytically, and for the second 
example, the state equation was solved numerically using an independent FEM solver (FEniCS [14]). Within the described gradient-descent method, we used a custom solver based on linear finite elements on a triangular mesh. We employed a line search according to the Armijo rule, although a minimum step length $h_{\min }$ was enforced in order to prevent the algorithm from getting "stuck" too early.

We used $D=[-1.5,1.5]^{2} \subset \mathbb{R}^{2}$ as hold-all domain for the level-set functions, which was discretised with 500 grid points in each space dimension. For the level-set time evolution, we used the described Fast Marching procedure based on Theorem 3. Everything is implemented in $\mathrm{C}++$ and GNU Octave [15]. The calculations were performed on a system with an amd64 quad-core processor. We used the multiple cores to run simulations for different regularisation parameters $\alpha$ in parallel and to try multiple step lengths for the line search simultaneously in the two examples, respectively, to make use of the available multi-threading capabilities.

Making use of parallel line search in this way, a typical descent step takes around $10 \mathrm{~s}$ for this already relatively fine spatial discretisation. (A particular timing for 2,000 descent steps is 20,203 s.) Most of this time is spent in the FEM solver, our level-set routines use only a minor fraction of the total processing time (for a particular profiling example, it was measured as $4.3 \%$ of the total time). This confirms that the Fast Marching Method is really suited to efficiently propagate geometries in time using the level-set method.

\section{A. The Effect of Different Regularisation Parameters}

For the choice of

$$
u_{d}(x, y)=\left(x^{2}+y^{2}\right)-\frac{1}{3}\left(x^{2}+y^{2}\right)^{3}
$$

and $\Omega_{\mathrm{ex}}=B_{1}(0,0)$ as the unit circle, a forcing function $f$ can be trivially computed from (5) such that $u_{d}$ is a solution. On $\Omega_{\mathrm{ex}}$, it satisfies also the boundary conditions. We applied $10 \%$ of Gaussian noise to $u_{d}$ and tried to recover $\Omega_{\mathrm{ex}}$ using the shape optimisation procedure for different regularisation parameters $\alpha$. Additional choices were $\beta=1, h_{\text {min }}=10^{-6}$ and $B=\left\{(x, y) \in \mathbb{R}^{2}|| x \mid<\frac{1}{2}\right.$ and $\left.|y|<\frac{1}{2}\right\}$. The result after 1,000 descent steps is shown in Figure 1. Figure 1a shows the difference between the calculated result and $\Omega_{\mathrm{ex}}$ as (normalised) $L^{1}$-difference of the corresponding signed distance functions. Figure $1 \mathrm{~b}$ shows how the numerical results (in colour) look like in comparison to the initial domain (black square) and $\Omega_{\mathrm{ex}}$ (black circle) for different values of $\alpha$. For this example, the regularisation ensures that the optimal shape is unique and can be found relatively well when the right choice of $\alpha$ is made. We have to admit, though, that this works particularly well here since regularisation via the boundary length favours the shape $\Omega_{\mathrm{ex}}$ we look for. One can easily see the typical behaviour of regularisation: With $\alpha=0$, the shape is not unique and the desired one is not found (although the cost is still minimised). With too large values of $\alpha$, the problem is distorted. This leads to a circle that is too small.

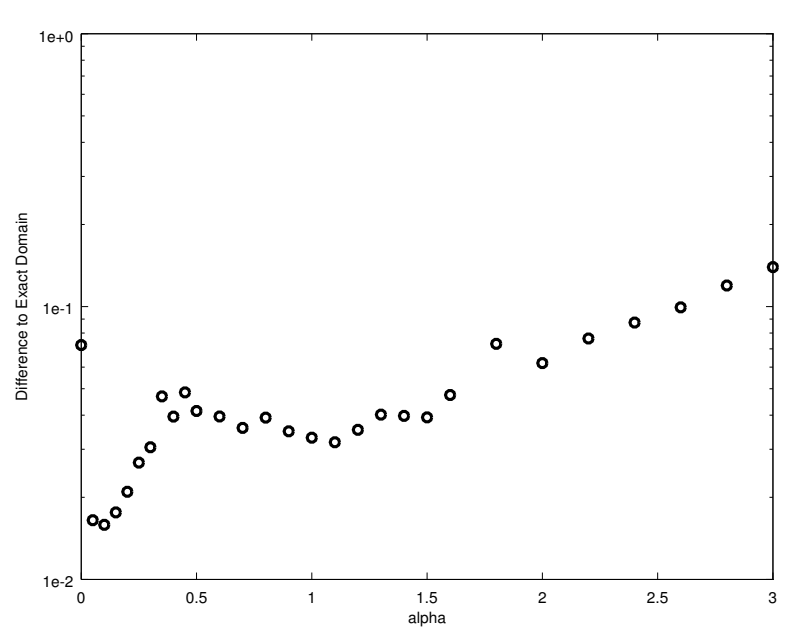

(a)

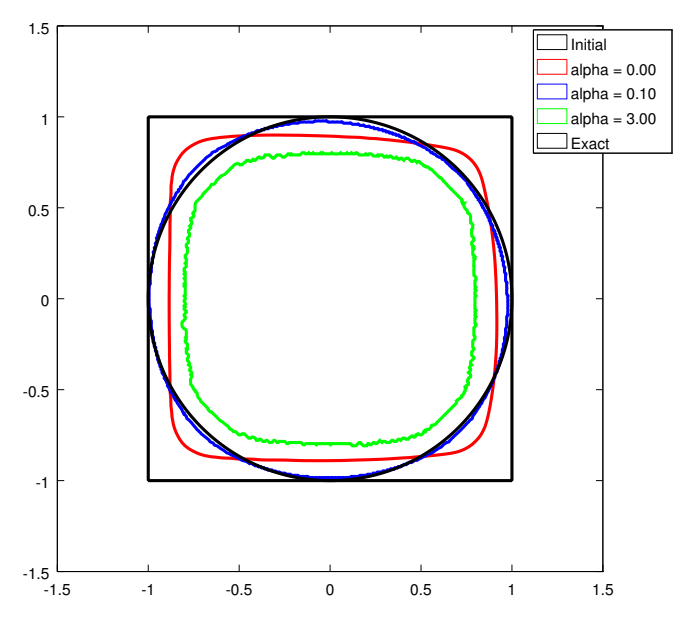

(b)

Fig. 1. Behaviour of the shape optimisation procedure for different regularisation parameters $\alpha$. See Subsection IV-A.

\section{B. Test for the Convergence Properties}

As the second example, we used a more complex geometry. The "exact domain" was chosen as

$$
\Omega_{\mathrm{ex}}=B_{1}(0,0) \backslash \overline{B_{\frac{1}{3}}(0.2,-1 / 3)}
$$

with a forcing function that penalises the "hole" strongly,

$$
f(x, y)=\min (0,10 y)+1000 \cdot \chi_{B_{\frac{1}{4}}(0.2,-1 / 3)}(x, y) .
$$

The observation domain was again rectangular, with

$$
B=\left\{(x, y) \in \mathbb{R}^{2}|| x \mid<\frac{1}{2} \text { and } y \in[0.1,0.5]\right\} \text {. }
$$

We used $h_{\min }=10^{-3}$ and applied neither regularisation ( $\alpha=0$ ) nor noise. Thus, the results show the behaviour of our method purely from an optimisation point of view rather than for inverse problems. We performed 1,000 descent steps 


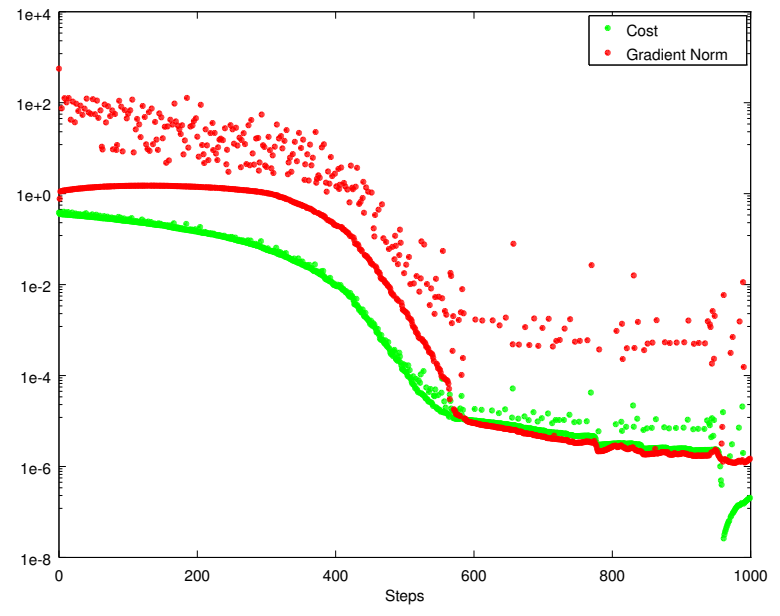

(a) $\beta=0.01$

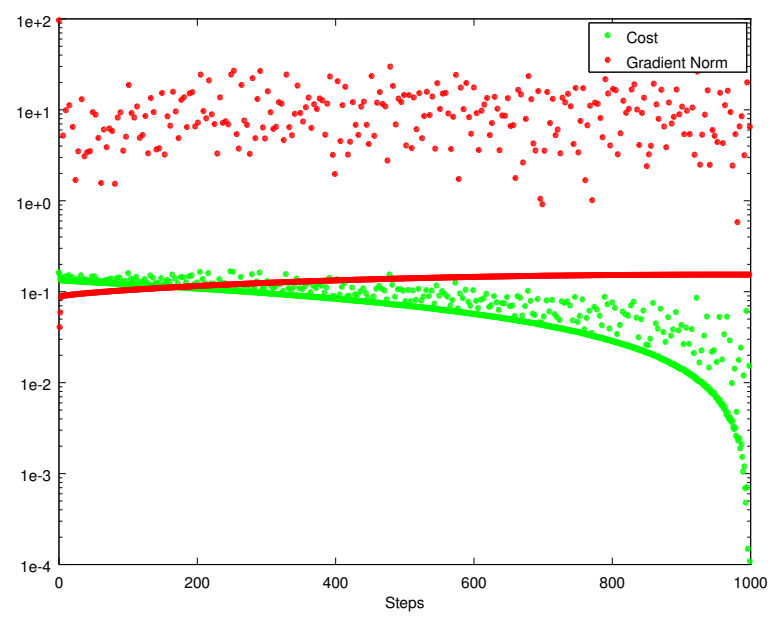

(b) $\beta=1$

Fig. 2. Decrease in the cost and gradient norm $\left(\|F\|\right.$ in the $H^{1}$-sense weighted by the chosen $\beta$ ) for the example problem described in Subsection IV-B.

for this example. Figure 2 shows how the cost and gradient norm decrease with the number of descent steps taken for the case of small and large $\beta$. Figure 3 shows how the resulting geometries look like. While the final geometry does not match $\Omega_{\mathrm{ex}}$ very much, it can be seen nicely that the cost as well as the gradient norm are still decreased almost to zero. This is due to the fact that the inverse problem is ill-conditioned and a lot of alternative shapes exist that result in almost the same minimal cost. Finally, one can also see that $\beta \ll 1$ leads to faster convergence in this example.

\section{ACKNOWLEDGEMENT}

The author would like to thank his $\mathrm{PhD}$ supervisor Wolfgang Ring of the University of Graz for fruitful discussions about these ideas as well as initially posing the research questions considered here. This work is supported by the Austrian

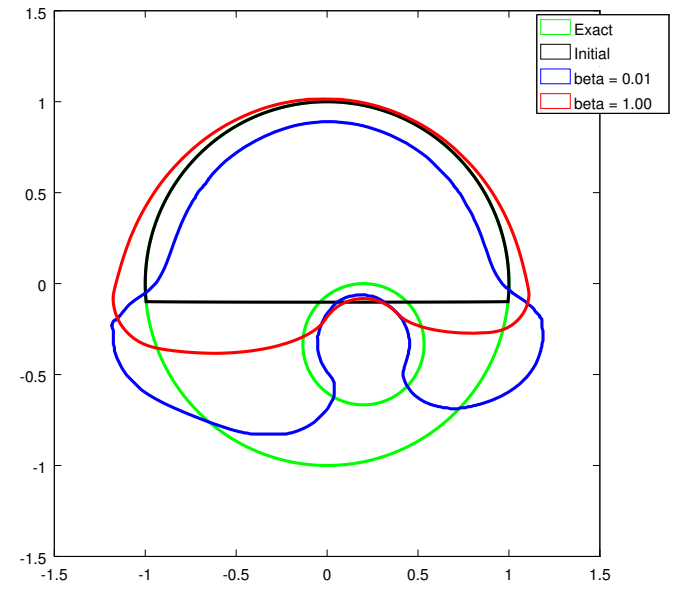

Fig. 3. The resulting domains for the example in Subsection IV-B in comparison to the initial geometry (black) and $\Omega_{\mathrm{ex}}$ (green).

Science Fund (FWF) and the International Research Training Group IGDK 1754.

\section{REFERENCES}

[1] M. C. Delfour and J.-P. Zolésio, Shapes and Geometries, 2nd ed., ser. Advances in Design and Control. SIAM, 2001.

[2] J. Sokolowski and J.-P. Zolésio, Introduction to Shape Optimization: Shape Sensitivity Analysis, ser. Springer Series in Computational Mathematics. Springer, 1992.

[3] J. Haslinger and R. A. E. Mäkinen, Introduction to Shape Optimization: Theory, Approximation, and Computation, ser. Advances in Design and Control. SIAM, 2003.

[4] S. Osher and J. A. Sethian, "Fronts propagating with curvaturedependent speed: Algorithms based on hamilton-jacobi formulations," Journal of Computational Physics, vol. 79, pp. 12-49, 1988.

[5] Y. Giga, Surface Evolution Equations: a level set approach, ser. Monographs in mathematics. Birkhäuser, 2006.

[6] G. Barles, H. M. Soner, and P. E. Souganidis, "Front propagation and phase field theory," SIAM J. Control and Optimization, vol. 31, no. 2 pp. 439-469, March 1993.

[7] M. G. Crandall, "Viscosity solutions: a primer," in Viscosity Solutions and Applications, ser. Lecture Notes in Mathematics. Springer, 1995

[8] M. G. Crandall, H. Ishii, and P.-L. Lions, "User's guide to viscosity solutions of second order partial differential equations," Bulletin of the American Mathematical Society, vol. 27, no. 1, pp. 1-67, July 1992.

[9] L. C. Evans, Partial Differential Equations, ser. Graduate Studies in Mathematics. American Mathematical Society, 1999.

[10] P.-L. Lions, Generalized solutions of Hamilton-Jacobi equations, ser Research Notes in Mathematics. Pitman Advanced Publishing Program, 1982.

[11] I. Capuzzo-Dolcetta, "A generalized hopf-lax formula: Analytical and approximations aspects," in Geometric Control and Nonsmooth Analysis, ser. Series on Advances in Mathematics for Applied Sciences, F. Ancona, Ed. World Scientific, 2008, vol. 76, pp. 136-150.

[12] J. A. Sethian, "A fast marching level set method for monotonically advancing fronts," Proceedings of the National Academy of Sciences, vol. 93, no. 4, pp. 1591-1595, 1996.

[13] L. C. Evans and R. F. Gariepy, Measure Theory and Fine Properties of Functions, ser. Studies in Advanced Mathematics. CRC Press, 1992.

[14] A. Logg, K.-A. Mardal, G. N. Wells et al., Automated Solution of Differential Equations by the Finite Element Method. Springer, 2012.

[15] J. W. Eaton, D. Bateman, and S. Hauberg, GNU Octave version 3.0.1 manual: a high-level interactive language for numerical computations. CreateSpace Independent Publishing Platform, 2009, ISBN 1441413006. [Online]. Available: https://www.gnu.org/software/octave/doc/interpreter 